\title{
Cannabidiol enhancement of exposure therapy in treatment refractory patients with phobias: study protocol of a randomized controlled trial
}

Febe E. van der Flier ${ }^{1,2,3,4}$ (D), Caroline M. B. Kwee ${ }^{2}$, Danielle C. Cath ${ }^{2,5,6}$, Neeltje M. Batelaan², Lucianne Groenink Puck Duits ${ }^{2}$, Date C. van der Veen ${ }^{4}$, Anton J. L. M. van Balkom ${ }^{3}$ and Johanna M. P. Baas ${ }^{1 *}$

\begin{abstract}
Background: Phobic anxiety disorders are among the most prevalent psychiatric disorders and are burdensome in terms of loss of quality of life and work productivity. Evidence-based treatments are relatively successful in the majority of patients, especially exposure therapy. However, a substantial subset of patients fails to achieve or stay in remission. Preclinical and genetic research have yielded evidence that the cannabinoid system is involved in the extinction of fear, presumed to underlie the beneficial effects of exposure therapy in phobic disorders. A cannabinoid constituent that may enhance endocannabinoid signaling is cannabidiol (CBD), a non-psychoactive component of cannabis. Hence, the addition of CBD to exposure therapy is expected to strengthen effects of treatment. To determine the added benefit of CBD on exposure therapy, we conduct a randomized controlled trial, in which patients in whom previous treatment as usual has not yielded sufficient response receive either CBD or placebo preceding 8 exposure sessions in a double-blind fashion. A subsidiary aim is to explore which (combination of) clinical, behavioral and genetic profiles of patients are related to treatment response.
\end{abstract}

Methods/design: This is an 8-week multicenter, randomized, double-blind, placebo-controlled trial. Seventy-two patients with social phobia or panic disorder with agoraphobia with incomplete response to earlier treatment will be included from outpatient clinics in the Netherlands. Patients are randomized to augmentation of exposure therapy with $300 \mathrm{mg}$ CBD or placebo. The study medication is administered orally, $2 \mathrm{~h}$ preceding each of the eight 90 min exposure sessions. Measurements will take place at baseline, first administration of medication, every session, mid-treatment, last administration of medication, post-treatment and at 3 and 6 months' follow-up. The primary outcome measure is the score on the Fear Questionnaire (FQ). In addition, determinants of the expected treatment enhancing effect of CBD will be explored.

Discussion: This is the first trial to investigate whether the addition of CBD to exposure therapy is effective in reducing phobic symptoms in treatment refractory patients with social phobia or panic disorder with agoraphobia.

Trial registration: Netherlands Trial Register NTR5100. Registered 13 March 2015. Protocol version: issue date 17 Jan 2018, protocol amendment number 7.

Keywords: Anxiety disorders, Panic disorder with agoraphobia, Social phobia, Cannabidiol, Exposure therapy, Randomized controlled trial, Cannabinoid system, Treatment resistance

\footnotetext{
* Correspondence: j.m.p.baas@uu.nl

${ }^{1}$ Department of Experimental Psychology and Helmholtz Institute, Faculty of

Social and Behavioural Sciences, Utrecht University, Utrecht, The Netherlands

Full list of author information is available at the end of the article
}

(c) The Author(s). 2019 Open Access This article is distributed under the terms of the Creative Commons Attribution 4.0 International License (http://creativecommons.org/licenses/by/4.0/), which permits unrestricted use, distribution, and

reproduction in any medium, provided you give appropriate credit to the original author(s) and the source, provide a link to the Creative Commons license, and indicate if changes were made. The Creative Commons Public Domain Dedication waiver (http://creativecommons.org/publicdomain/zero/1.0/) applies to the data made available in this article, unless otherwise stated. 


\section{Background}

Phobic disorders (e.g. social anxiety disorder, panic disorder with agoraphobia) are among the most prevalent disorders according to the World Health Organization's World Mental Health Survey Initiative [1]. These and other anxiety disorders have major impact on health, individual suffering and societal costs [2]. The estimated societal costs in Europe as a result of anxiety disorders were 74.4 billion Euros in 2010, affecting more than 69 million Europeans [3]. Anxiety disorders often co-occur with other mental health disorders $[4,5]$, and are associated with an increased risk of suicide [6]. Spontaneous recovery from these disorders is uncommon; if left untreated, phobias typically follow a chronic course, with low remission and high relapse rates [7].

The current evidence-based treatment entails exposure with response prevention therapy, either alone or in combination with serotonin reuptake inhibitors (SSRIs). Exposure therapy is relatively successful, with improvement in up to $60 \%$ of patients. However, only 30 to $50 \%$ of phobic patients achieves full remission [8]. Likewise, treatment with SSRIs is relatively effective, however, many patients experience relapse after discontinuing SSRI treatment $[9,10]$, while the effects of successful exposure treatment seem to be more sustainable [11]. Considering the high prevalence of anxiety disorders and the large number of patients for whom the anxiety symptoms remain refractory after (repeated) gold-standard treatments, new approaches to the treatment of anxiety are urgently needed $[12,13]$. Preclinical as well as clinical studies have pointed to the relevance of utilizing fear learning paradigms for a deeper understanding of the neurocircuitry and neurochemistry of the fear system involved in anxiety disorders [14]. Specifically, patients with anxiety disorders show stronger fear responses during extinction than comparison subjects [15], and poor fear extinction is predictive of poor outcome in exposure therapy [16].

A potential novel target for the facilitation of fear extinction has been derived from preclinical research. The crucial involvement of the cannabinoid system in fear extinction was first shown by Marsicano et al. [17]. The results show that (genetic or pharmacological) blockage of transmission at the cannabinoid receptor 1 (CB1) inhibits extinction of fear in mice. This is not surprising given the fact the CB1 receptors are richly expressed in memory-related brain areas such as hippocampus and prefrontal cortex, and as such can modulate (fear) memory [18]. In the last 15 years many studies have extended this finding using both animal and human subjects (for reviews see [12] or [19]). Animal research has shown that facilitation of the endocannabinoid system (ECS) enhances extinction, whereas blocking or deletion of $\mathrm{CB} 1$ receptors impairs extinction. In healthy human subjects we have demonstrated that genetic variation in a
CB1 polymorphism significantly affected extinction learning [20]. Furthermore, the administration of cannabinoids in humans has shown to strengthen extinction and protect against reinstatement of fear [21-23]. In summary, previous research clearly points to the ECS as a promising candidate for extinction enhancement. Until now, studies in humans have mainly investigated the effects of delta-9-tetrahydrocannabinol (THC), which has been shown to decrease physiological measures of fear during extinction [24] and recall [21]. However, THC is not suitable for phobic patients given the diversity of psychoactive effects caused by THC, among which the high that recreational users of cannabis seek.

In the meantime, studies have demonstrated the potential benefit of another important ingredient of cannabis: cannabidiol (CBD, for a review see [25]). CBD interacts with several receptors in the brain including cannabinoid receptors 1 and 2 , transient receptor potential vanilloid type 1 (TRVP1) and serotonin 1A (5-HT1A) receptor, and inhibits or in other ways negatively affects the function of the enzyme that degrades endogenously released cannabinoid neurotransmitters (fatty acid amine hydrolase; FAAH [26]). In line with FAAH's function in degrading anandamide [27], inhibition of FAAH has been shown to increase levels of anandamide. Preclinical research indicates that $\mathrm{CBD}$ enhances fear extinction and reconsolidation, and co-administrating CB1 antagonists block such effects suggesting that they are exerted via modulation of the ECS [28, 29]. Extinction of conditioned fear is proposed to underlie the effect of exposure therapy [14]. Hence, the finding that CBD specifically affects (the consolidation of) extinction suggests a potential use of CBD in augmenting the effect of exposure therapy. This leads to the hypothesis that administration of CBD during sessions of exposure therapy is expected to specifically enhance the extinction of pathological fears. The advantage of this application is that CBD needs to be administered occasionally, i.e. preceding exposure sessions only.

We aim to take this previous research to the next level by conducting the first randomized controlled trial with CBD versus 7, administered in a double-blind fashion, for the augmentation of exposure treatment in patients with social phobia or panic disorder with agoraphobia. Also, we aim to specifically target patients who have already received one of the gold-standard treatments without responding sufficiently or having relapsed, because this group needs additional approaches most.

The main study aim is to test whether administration of CBD as an augmentation step in exposure therapy can strengthen treatment outcome in patients with phobic disorders who have previously failed to respond satisfactorily to evidence-based treatment. Clinical measurements are used to investigate whether the effect of CBD on exposure is quicker, stronger, or longer-lasting than regular exposure 
therapy only. Additionally, there are various exploratory subsidiary aims in this study. First, a fear conditioning and extinction task is applied at baseline. This task has shown enhanced fear responses in patients with anxiety disorders as opposed to healthy comparison subjects [30]. This task also revealed different extinction trajectories, with patients being overrepresented in a poor extinction profile [16]. These profiles have also shown to be sensitive to differences between patients who will benefit from exposure treatment and those who will not. A re-extinction assessment is done after the first medication administration. The aim of this task is to explore a) whether patients with a specific profile can particularly benefit from CBD augmentation during exposure, and b) the acute effects of CBD intake on fear extinction. Second, we aim to explore the interactions between specific genetic variation and CBD administration on treatment effect. We are particularly interested in studying whether variants within the cannabinoid receptor 1 gene are involved in a differential response to CBD augmented exposure therapy, including rs2180619 identified in our previous study in healthy individuals associated with impaired spontaneous extinction of conditioned fear [20]. Additionally, impact of genetic polymorphisms within the FAAH gene [31] and genetic polymorphisms identified as being related to treatment response in anxiety disorders [32] will be explored. Similarly, clinical predictors of treatment response will be assessed to determine which sort of patients might benefit most from this augmented treatment. Lastly, we aim to assess cost-effectiveness of CBD enhancement of exposure treatment.

\section{Methods}

\section{Study design}

The study encompasses a multi-site randomized, double-blind, placebo-controlled fixed dose clinical trial for patients with treatment resistant social phobia or panic disorder with agoraphobia. Either placebo $(N=36)$ or $300 \mathrm{mg}$ cannabidiol $(\mathrm{N}=36)$ will be administered 8 times as an adjunct to 8 weekly 90 min sessions consisting of standardized exposure therapy. The study has been approved by the Medical Ethics Committee of the University Medical Centre Utrecht. Written informed consent will be obtained from all participants. The enrollment of the first participant was on 15 February 2016, recruitment is ongoing at the time of submission. Figure 1 displays a flowchart of the study.

\section{Participants \\ Inclusion criteria}

- Adult patients between 18 and 65 years with a phobic disorder (social phobia or panic disorder with agoraphobia), diagnosed with the Structured Clinical Interview on DSM-IV disorders (SCID; [33])

- At least one attempt to treat the disorder, according to guidelines, either by means of psychotherapy or with the use of serotonergic antidepressants, has induced insufficient clinically relevant response during or after treatment

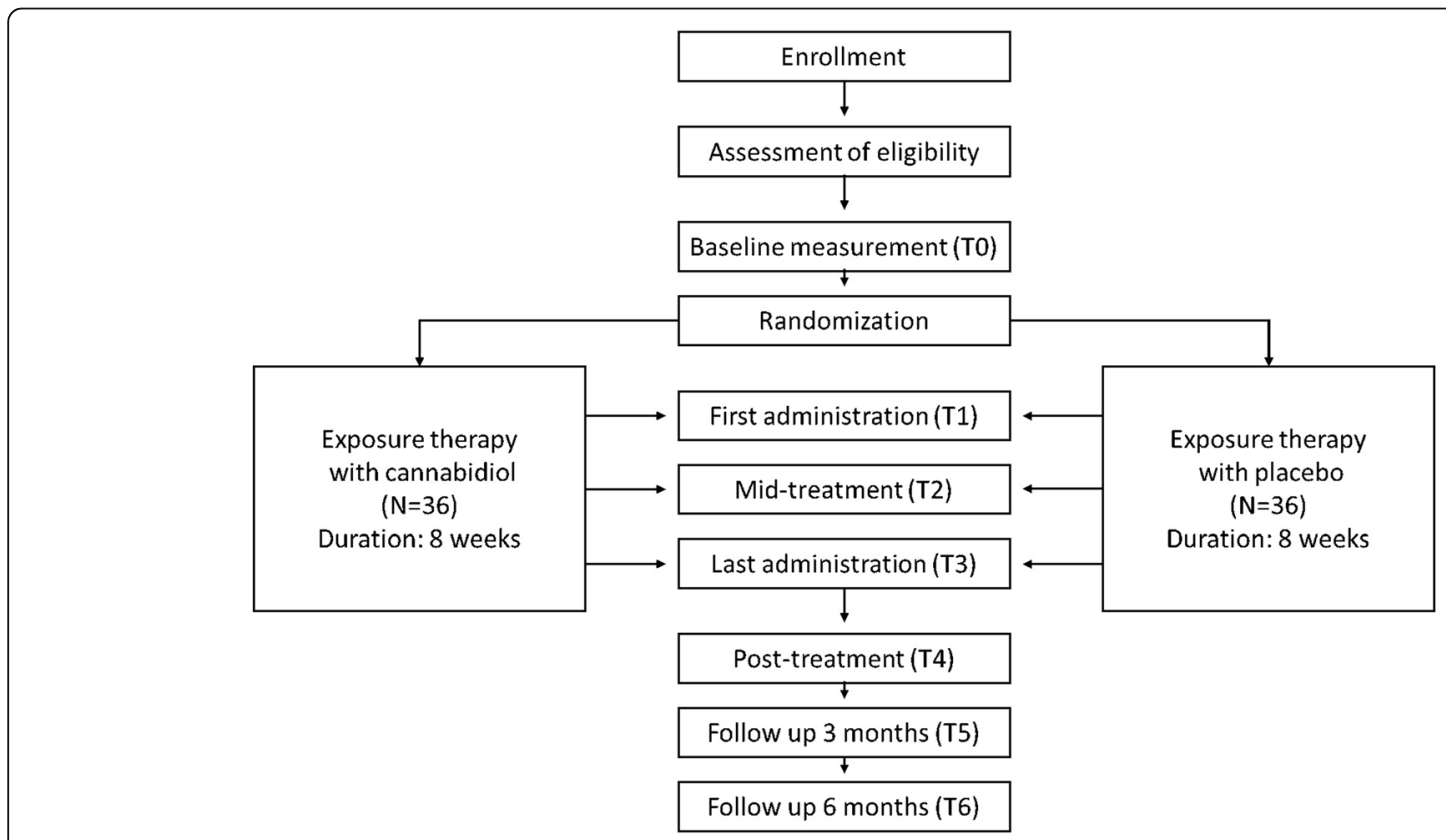

Fig. 1 Flowchart of the study design. Data is collected both during T0-T6 measurements and therapy sessions, see Table 1 for a complete overview 


\section{Exclusion criteria}

- Co-morbid psychiatric disorders, i.e. current severe major depressive or bipolar disorder, psychosis, dependence on alcohol and drugs, as determined by the SCID

- Mental deficiency (IQ < 80, as determined by the Nederlandse Leestest voor Volwassenen (NLV; [34]))

- Autism traits (AQ > 32, as determined by the Autism Spectrum Quotient (AQ; [35]))

- Inadequate proficiency in Dutch, both verbal and written

- (A history of) epilepsy, brain damage, cardiac, renal or liver abnormalities

- History of allergies to medication (adverse reactions or rash)

- Use of antipsychotic medication

- Regular daytime use of benzodiazepines, since use concomitant to exposure has been shown to hamper the treatment effect [36]

- Changes in dosing regimen of serotonergic antidepressants shorter than 4 weeks prior to study entry (i.e. use of serotonergic antidepressants at a stable regimen throughout the study is permitted)

- Use of recreational drugs (among others THC, XTC, cocaine) from 2 months preceding study entry

- Pregnancy or breastfeeding

\section{Sample size}

The CBD and placebo groups will each include 36 patients. The sample size is aimed at detection of a Cohen's D effect size of 0.6 , based on effect sizes found in previous published studies on the augmentation of exposure with $d$-cycloserine [37]. This sample size has been calculated using G*"power version 3.0.10 [38], with a repeated measures design for two groups with two measurements, an envisioned effect size of 0.6 Cohen's D, error probability of 0.05 , power of 0.8 and correlation among repeated measurements of 0.6 based on previous clinical data.

\section{Recruitment}

Patients will be recruited at anxiety outpatient clinics of specialized mental health care centers in the Netherlands (Altrecht, GGZinGeest and UCP). Before including patients in the study, they first undergo an intake interview by an experienced therapist. Eligible patients are informed about the study and are invited to a screening and diagnostic interview (SCID) by the researcher or a trained research assistant to confirm inand exclusion criteria. Patients receive the information brochure and informed consent form if they are eligible and interested in participating. Informed consent is obtained by the researcher or a trained research assistant before the start of the baseline assessment. Additionally, participants can opt to consent to the use of their genetic material in larger international databases.

\section{Randomization and blinding}

The randomization (CBD or placebo) is conducted by an independent statistician using a computer algorithm, stratifying for study location and diagnosis (panic disorder with agoraphobia or social phobia). Patients are allocated to one of the medication groups after baseline measurements according to the order of patients in the stratum. Investigators, research assistants, therapists and participants will be blinded with respect to randomization. The capsules containing the different medications are identical in appearance except for filling which is either CBD or lactose (placebo). An independent data manager can break the randomization code in case of pregnancy, allergic reactions or any severe inexplicable symptoms. Apart from these circumstances, unblinding will not be done until after the last patient has completed the last follow up measurement.

\section{Intervention}

Eight 90-min exposure sessions will be carried out by therapists who are well trained in cognitive behavioral therapy (CBT), including exposure exercises, and in the current study protocol. Protocols in this study are based on standardized protocols of exposure with response prevention in social phobia [39] and in panic disorder with agoraphobia [40]. The protocols consist of therapist-assisted exposure in vivo to fear-provoking thoughts and situations, coupled with response prevention treatment (e.g. not leaving the feared situation or using safety behaviors), tailored to idiosyncratic symptoms of the patients. After every therapy session homework is given, resulting in patients doing at least 8 exposure exercises per week.

Two hours prior to the exposure treatment sessions the study medication is administered. Timing of administration is based on a study by Englund et al. [41], indicating Tmax at $3 \mathrm{~h} 45 \mathrm{~min}$ after administration with high plasma levels from $2 \mathrm{~h}$ onwards. Therefore, taking the medication $2 \mathrm{~h}$ before the start of the session results in relatively stable CBD levels during the entire session.

The eight sessions that are part of the study protocol are not expected to be sufficient for most patients to achieve remission, but this allows sufficient room to investigate whether $\mathrm{CBD}$ strengthens therapy response relative to placebo. After the eight sessions in the study protocol patients can continue treatment as needed without further administration of study medication.

\section{Assessments}

Response to treatment will be assessed at baseline (T0), at mid-treatment (T2), post-treatment (T4) and at 3 and 6 months' follow-up (T5 and T6 respectively). During 
treatment, a short assessment is done at each therapy session. Table 1 provides an overview of the measures that are used at each time point. The primary endpoint of this study is the clinical outcome post-treatment (at assessment T4). The other measurements are aimed at the time course of the effect. The mid-treatment and per session assessments are specifically aimed at examining the possibility of a quicker and/or stronger effect of exposure with CBD as opposed to placebo, whereas the follow up measurements allow evaluation of potential long term beneficial effects of CBD. Furthermore, preceding the first and last treatment session with medication administration (T1 and T3 respectively) several secondary measures will be used to study the mechanism underlying acute effects of CBD. Also, blood samples from these assessments will be used to determine CBD plasma levels.

\section{Outcome measures}

\section{Primary outcome}

The primary outcome measure is the Fear Questionnaire (FQ; [42]) which will be administered at every time point (T0-T6) and at every treatment session.
The $F Q$ is a part of a standard self-report questionnaire measuring avoidance, the complete form also includes one specific main target phobia, a global phobia rating, and five associated anxiety and depression symptoms (not included in this study). The version of the FQ employed here consists of 15 items asking about the most common phobias rating avoidance using a nine-point scale from ' 0 : would not avoid it' to '8: always avoid it'. The score reflects the level of avoidance, with a total score range from 0 to 120 . Three subscores can also be derived using the sum of 5 items, concerning Agoraphobia, Blood injury phobia and Social phobia.

\section{Secondary outcomes}

Clinical questionnaires Various secondary outcome measures are used to further explore the effect of CBD augmentation on general clinical and specific disorder-related symptoms. Baseline scores on these questionnaires will be used to develop clinical determinants of the effect from augmentation with CBD. All secondary clinical questionnaires are administered at baseline, mid- and post-treatment and follow up assessments.

Table 1 Overview of assessments

\begin{tabular}{|c|c|c|c|c|c|c|c|c|c|}
\hline Measure & Assessment & T0 & $\mathrm{T} 1$ & $\mathrm{~T} 2$ & T3 & T4 & T5 & T6 & Treat-ments \\
\hline SCID & Diagnosis & $x$ & & & & & & & \\
\hline General patient characteristics & Demographic information & $x$ & & & & & & & \\
\hline Clinical background & Therapy history & $x$ & & & & & & & \\
\hline CTQ & Childhood trauma & $x$ & & & & & & & \\
\hline AQ & Autism quotient & $x$ & & & & & & & \\
\hline $\mathrm{FQ}$ & Presence and severity phobic symptoms & $x$ & & $x$ & & $x$ & $x$ & $x$ & $x$ \\
\hline BAl & Anxiety severity & $x$ & & $x$ & & $x$ & $x$ & $x$ & $x$ \\
\hline CGl & Clinical global impression & & & & & & & & $x$ \\
\hline SUDS & Degree of habituation and extinction & & & & & & & & $x$ \\
\hline BDI & Depression & $x$ & & $x$ & & $x$ & $x$ & $x$ & \\
\hline BSQ & Somatic symptoms & $x$ & & $x$ & & $x$ & $x$ & $x$ & \\
\hline EQ5D & Quality of life & $x$ & & $x$ & & $x$ & $x$ & $x$ & \\
\hline Tic-P & Loss of work and productivity plus health care costs & $x$ & & $x$ & & $x$ & $x$ & $x$ & \\
\hline SPAI & Social phobia and anxiety severity & $x$ & & $x$ & & $x$ & $x$ & $x$ & \\
\hline LSAS & Social anxiety severity (SOC PHOB) & $x$ & & $x$ & & $x$ & $x$ & $x$ & \\
\hline PDSS & Panic disorder severity (PD + AGO) & $x$ & & $x$ & & $x$ & $x$ & $x$ & \\
\hline Ml & Mobility inventory (PD + AGO) & $x$ & & $x$ & & $x$ & $x$ & $x$ & \\
\hline ACQ & Agoraphobia severity (PD + AGO) & $x$ & & $x$ & & $x$ & $x$ & $x$ & \\
\hline Fear conditioning task & Acquisition and extinction of fear learning & $x$ & $x$ & & $x$ & & & & \\
\hline Blood & CBD level, DNA, epigenetics & & $x$ & & $x$ & & & & \\
\hline
\end{tabular}

Questionnaires that are only assessed for a specific diagnosis are specified between brackets (panic disorder with agoraphobia $=$ PD + AGO, social phobia $=$ SOC $\mathrm{PHOB}) . \mathrm{T} 0=$ Baseline, $\mathrm{T} 1=$ First medication administration, $\mathrm{T} 2=$ Mid treatment, $\mathrm{T} 3=$ Last medication administration, T4 = Post treatment, T5 = Follow up (3 months), T6 = Follow up (6 months), Treatments = All 8 therapy sessions. SCID Structured Clinical Interview for DSM disorders axis I, CTQ Childhood Trauma Questionnaire, $A Q$ Autism spectrum Quotient, FQ Fear Questionnaire, BAI Beck Anxiety Inventory, CGI Clinical Global Impression, SUDS Subjective Units of Distress Scale, BDI Beck Depression Inventory, BSQ Bodily Sensations Questionnaire, EQ5D EuroQol, Tic-P Trimbos and iMTA questionnaire on Costs associated with Psychiatric illness, SPAI Social Phobia and Anxiety Inventory, LSAS Liebowitz Social Anxiety Scale, PDSS Panic Disorder Severity Scale, MI Mobility Inventory, ACQ Agoraphobic Cognitions Questionnaire 
The Beck Anxiety Inventory (BAI; [43]) is a 21-item self-report instrument that assesses the overall severity of anxiety. Respondents rate how much each symptom bothered them the past week on a 4-point scale, ranging from 0 (not at all) to 3 (severely, I could barely stand it). The BAI is scored by summing the ratings for all the 21 symptoms to obtain a total score ranging from 0 to 63 . Whereas avoidance (measured using the $\mathrm{FQ}$ ) is a highly relevant clinical construct, restricting analysis to just this aspect may overlook impact on other symptoms of anxiety, such as physiological changes, that may not have a direct effect on behavior as measured by the FQ. Therefore, we have chosen to use the BAI as most important secondary outcome, which is why it is also administered at every treatment session with the FQ.

The Beck Depression Inventory-II (BDI-II; [44]) is a 21-item self-report instrument that is the most widely used to assess the presence and/or intensity of depressive symptoms. Similar to the BAI, symptoms are scored on a 4-point scale resulting in total scores ranging from 0 to 63.

The Body Sensations Questionnaire (BSQ [45]) is a 17-item self-report instrument assessing fear for bodily sensations associated with autonomic arousal. Items are rated on a 5-point scale, total scores range from 17 to 85 .

The Social Phobia and Anxiety Inventory (SPAI; [46]) is used to assess specific somatic symptoms, cognitions and behavior across a range of potentially fear-producing situations. The original SPAI has two subscales, Social phobia (32 items) and Agoraphobia (13 items). A shorter SPAI-18 has been developed assessing only the Social Phobia scale [47]. In this study the SPAI-18 is combined with the original Agoraphobia subscale, resulting in 31 items. Thirteen items require separate ratings concerning either four different social situations or physiological and cognitive questions. Mean scores are calculated for these items based on the 7 -point scale ranging from 1 (never) to 7 (always). To obtain the score the number of items is subtracted from the summed item scores. The maximum score for the SPAI-18 is 108, and for the Agoraphobia scale 78.

Only during the treatment sessions the Clinical Global Impression (CGI; [48]) and Subjective Units of Distress (SUDS; [49]) are administered. The CGI consists of 2 items, measuring illness severity and improvement. The items are rated on a 7-point scale by the therapist, with the severity scale ranging from 1 (normal) to 7 (amongst the most severely ill patients), and the improvement scale ranging from 1 (very much improved) to 7 (very much worse). Each component is rated separately, there is no total score [50]. The SUDS are used during exposure to measure within-session extinction. Before and after the exposure in vivo exercise percentage of fear and credibility of thoughts about the exercise are rated by the patient [51].
Besides the broader clinical questionnaires, diagnosis-specific questionnaires are only administered to patients with the diagnosis in question. Questionnaires pertaining to the diagnosis of panic disorder with agoraphobia:

The Panic Disorder Severity Scale (PDSS; [52]) is a 7-item clinician-administered instrument assessing severity of panic disorder and monitoring treatment outcome. Items are rated on a 5-point scale which ranges from 0 to 4 , total scores are calculated by summing the scores for the items resulting in a range of 0 to 28 .

The Mobility Inventory (MI; [53]) is a 27-item self-report instrument for the measurement of agoraphobic avoidance behavior in specific situations. These situations are rated both when patients are accompanied and when they are alone. Items are rated on a 5-point scale which ranges from 1 (never) to 5 (always), the score is calculated by averaging the items.

The Agoraphobic Cognitions Questionnaire (ACQ; [45]) is a 14-item self-report instrument assessing thought concerning negative consequences of experiencing anxiety. Each item is rated on a 5-point scale ranging from 1 (never occurs) to 5 (always occurs), total scores are calculated by averaging the items. Specifically, catastrophic thoughts typically noted during exposure to anxiety-provoking experiences are used, making it highly relevant for the assessment of therapy success.

Questionnaire pertaining to the diagnosis of social phobia:

The Liebowitz Social Anxiety Scale (LSAS; [54]) is a self-report instrument with 24 items measuring both fear and avoidance across a number of social situations. Fear scale ratings range from 0 (no fear) to 3 (severe fear), avoidance ratings also range from 0 to 3 and are based on percent of time avoiding the situation $(0=$ never, $1=$ occasionally (10\%), $2=$ often (33-67\%), and $3=$ usually (67-100\%). The LSAS is divided in two subscales, related to performance anxiety (11 items) and social interaction (13 items).

All clinical questionnaires have been shown to have adequate reliability and validity (ACQ [55], BAI [56], BDI [57], BSQ [58], FQ [59], LSAS [60], MI [53], PDSS [61], SPAI [47]), except for the CGI [62] which is advised to be used in accordance with other validated questionnaires, which are used in this study.

General patient characteristics Demographic information such as age, gender, education, employment, nationality and marital status will be collected using a general demographic questionnaire at baseline. Current use of drugs and medication is assessed with a short questionnaire. Additional questions are asked concerning the clinical background, e.g. treatment history, and the Childhood Trauma Questionnaire [63] is administered. 
Experimental assessment of fear learning To explore whether capacity for fear and extinction learning at baseline impacts the effect of CBD, and whether treatment with CBD has impact on improvements in extinction learning after treatment, an experimental fear conditioning and extinction task will be used to assess the capacity to acquire and extinguish conditioned fear [30]. At baseline, this task will investigate the acute effect of CBD on extinction learning, a second extinction phase with the same conditioned stimuli as at baseline is administered 2 $\mathrm{h}$ after the first ingestion of the medication. This additional fear extinction phase is administered 1 to 2 weeks after administration of the baseline fear conditioning task. Finally, post-treatment the same fear conditioning task will be administered, with minor adaptations to minimize previous learning effects (e.g. with different conditioned stimuli). With this post-treatment task, changes in rate of extinction due to treatment is compared between the $\mathrm{CBD}$ and placebo groups.

Genetics Profiling of phobic patients based on genetic variance will be done to examine potential factors that have impact on the effect of exposure therapy and on the effect of CBD augmentation. In general, we expect more benefit of CBD augmentation for individuals with genetic profiles associated with lack of spontaneous extinction. More specifically, for the impact on CBD augmentation, genetic variance in CNR1 [20], FAAH [31, 64] and genes related to treatment response in phobic disorders [32], will be analyzed.

Cost effectiveness The documentation of (non-)medical costs and productivity loss will be collected to assess cost-effectiveness of CBD-augmented psychotherapy. Both cost effectiveness-questionnaires are administered at baseline, mid- and post-treatment and follow up assessments.

The Treatment inventory of costs in Psychiatric patients (Tic-P [65]) is a self-report questionnaire consisting of two parts, medical resource, including volume of mental and general health care utilization (direct medical costs), travel to and from health care providers (non-medical costs), and productivity loss, generated by absence from paid work (indirect costs). Corresponding costs are calculated by multiplying the volumes by the corresponding reference unit prices [66].

The EuroQol five dimensions (EQ5D [67]) is a 5-item self-report instrument which is the most commonly used generic health status measurement. The items have five response categories from no problems to incapacity/extreme problems. Additionally, a visual analogue scale (VAS) is used to rate their health on a scale ranging from 0 (worst possible health) to 100 (best possible health).

\section{Statistical analysis}

\section{Treatment augmentation}

Data concerning the primary and secondary outcome measures will be analyzed by comparing the scores on the measurement scales using mixed modeling, with medication (CBD vs. placebo) and time (time points: baseline, mid-treatment, post-treatment and follow-ups). Analyses are conducted according to the intention-to-treat principle, i.e. all patients who have completed the baseline assessment are included in the analyses. Furthermore, also a 'completers only' analysis will be done including just the participants who have completed the treatment and participated in all measurements (T0-T6).

\section{Patient profiling}

To determine which patient characteristics may predict additional benefit of CBD augmentation, explorative multilevel analyses with treatment success as dependent variable will be performed, with the following independent variables (among others); medication (CBD or placebo), diagnosis (panic disorder with agoraphobia or social phobia), fear learning (response during extinction, and reduction of fear from acquisition to extinction), cannabinoid system genetics (using a candidate gene approach focused on CNR1 and FAAH), prior treatment history (failed CBT, SSRI, or both), clinical state at baseline and demographic variables (gender, age).

\section{Fear learning}

Acute effect of CBD on fear learning is analyzed with retention of conditioned fear, and rate of extinction in this re-extinction phase as outcome variables, and medication as independent variable. Impact of CBD-augmented exposure therapy on changes in rate of extinction from baseline between the CBD and placebo groups is examined by comparing extinction before and after treatment.

\section{Cost effectiveness}

Costs of illness and intervention is measured using resource utilization which will be valued with unit costs based on standardized real cost price calculations. The economic evaluation is primarily designed as exploratory cost-effectiveness analyses.

\section{Data management and dissemination}

To improve data completeness we have developed a study specific digital file to store personal information and to get reminders for upcoming assessments and missing data, which can be accessed by the researchers per participating center. Actual data are not collected in this file, but stored digitally in a database on the servers of GGZinGeest, separately from personal information of the participants. To ensure data quality and reliability, questionnaires are administered online and saved digitally, together with and data from interviews. Data from treatment sessions is 
collected and entered into the study data base and subsequently checked by research assistants. Data management and monitoring is conducted by data managers from GGZinGeest. Study conduct is reported and audited in interim, with final reports to the funding agency. The procedures comply with Dutch data privacy laws.

If participants wish to withdraw from the intervention, their participation in the post-treatment and follow up assessments are encouraged. Unless participants have withdrawn consent for follow-up, repeated attempts are made to contact participants. In a step-wise manner, this will involve sending emails and calling the individual on contact numbers provided on various days of the week and at different times. As much information as possible will be collected from protocol non-adherers.

Adverse events occurring after entry into the study are recorded. Investigators will determine relatedness of an event to the study drug based among others on temporal relationship and the subject's clinical course.

Any modifications to the protocol which may impact the conduct of the study, potential benefit of the patient or may affect patient safety, including changes of study objectives, study design, patient population, sample sizes, study procedures, or significant administrative aspects will require a formal amendment to the protocol. Such amendment will be approved by the Ethics committee prior to implementation and information on the Trial register website will be updated to ensure transparency.

There are no interim analyses planned. The final trial dataset will be accessible to the researchers and data managers. Results of the analyses will be published in scientific journals and presented on scientific conferences by the researchers, regardless of the outcome. A summary report of trial results written in lay language will be sent to study participants and other people who have expressed interest.

\section{Discussion}

Phobic disorders are among the most prevalent disorders and have a major impact on the life of patients and society as a whole resulting in suffering and associated costs. Evidence-based treatments of these disorders, while effective for a large number of patients, are not adequate for a substantial group who are not sufficiently relieved from their anxiety symptoms. One strategy may be to boost the effectiveness of current treatments. Enhancing exposure therapy with pharmacological agents that affect the neurological processes involved in the extinction of fear is an avenue that has been explored with augmentation using $d$-cycloserine, with mixed success [68]. Since an enhancer of exposure therapy is needed but the compounds so far have not proven to be sufficiently effective, we have opted to use a new strategy using the modulation of the endocannabinoid system. This study will be the first clinical trial in which cannabidiol is used to augment exposure therapy for phobic patients.
It is important to note that this study is investigator initiated, and independent from pharmaceutical or other industry interests. Findings will be submitted to a peer reviewed scientific journal for publication.

This study is based on the preclinical evidence that ECS manipulations can be used to enhance (the retention of) fear extinction. However, acute anxiolytic effects of cannabidiol have also been reported. One study reported anxiolysis during a public speaking challenge, which resembles the type of challenges that patients with phobia are faced with during exposure therapy [69]. Hence, an additional possible outcome of the study is that cannabidiol reduces fear and anxiety acutely during the treatment sessions, making the treatments easier to tolerate. Despite the conviction based on other anxiolytic treatments that anxiolysis during exposure reduces effectiveness [70], the expectation is that cannabidiol may combine acute anxiolysis with enhanced retention of treatment effects.

A strong feature of this study is the exploratory assessment of genetic, experimental and clinical differences between patients related to extinction and subsequent treatment response. The results of this study might give rise to new insights into the possibility of personalized treatment, by exploring whether this strategy is best, specifically for patients with certain characteristics.

\section{Abbreviations}

5-HT1A: Serotonin 1A; ACQ: Agoraphobic cognitions questionnaire; AQ: Autism spectrum quotient; BAl: Beck anxiety inventory; BDI: Beck depression inventory; BSQ: Bodily sensations questionnaire; CB1: Cannabinoid 1; CBD: Cannabidiol; CBT: Cognitive behavioral therapy; CGI: Clinical global impression;

CTQ: Childhood trauma questionnaire; ECS: Endocannabinoid system; EQ5D: EuroQol 5D; FAAH: Fatty acid amide hydrolase; FQ: Fear questionnaire; LSAS: Liebowitz social anxiety scale; MI: Mobility inventory; NLV: Nederlandse leestest voor volwassenen; PDSS: Panic disorder severity scale; SCID: Structured clinical interview on DSM-IV disorders; SPAI: Social phobia and anxiety inventory; SSRI: Selective serotonin re-uptake inhibitor; SUDS: Subjective units of distress scale; THC: Delta-9-tetrahydrocannabinol; Tic-P: Trimbos and iMTA questionnaire on Costs associated with Psychiatric illness; TRPV1: Transient receptor potential vanilloid type 1; VAS: Visual analogue scale

\section{Acknowledgements}

Not applicable.

\section{Funding}

This study is funded by ZonMw (project number 40-41200-98-9269). The funding agency has reviewed and approved the research plan, but had no role in the planning of the study design and will not be involved in data collection and analysis, decision to publish or preparation of the manuscript.

\section{Availability of data and materials}

The datasets used and/or analyzed during the current study from patients consenting to sharing their data are available from the corresponding author on reasonable request.

\section{Roles and responsibilities}

Trial sponsor: Department of Experimental Psychology, Helmholtz Institute, Utrecht University.

Representative: Prof. dr. J.L. Kenemans, j.l.kenemans@uu.nl

The sponsor has no role in the design of this study and will not have any role during its execution, analyses, interpretation of the data, or decision to submit results.

User committee. 
- Agreement of demands for patients.

- Agreement of patient recruitment procedure, information letter and consent form.

Data management.

- Maintenance of trial IT system and data entry.

\section{Authors' contributions}

$J B(P I), D C, A v B, L G$ and NB obtained funding for this study. All authors contributed to the design of the study. FvdF and CK coordinate the recruitment of patients and the data collection. JB, DC, AvB and NB are responsible for the overall design and supervision. FvdF wrote the manuscript. All authors (FvdF, JB, $D C, P D, D v d V, A v B, L G, N B, C K$ read, contributed and approved the final manuscript.

\section{Ethics approval and consent to participate}

The study has been approved by the Medical Ethics Committee of the University Medical Centre Utrecht (reference number: NL50898.041.15). Written informed consent will be obtained from all participants.

\section{Consent for publication}

Not applicable.

\section{Competing interests}

The authors declare that they have no competing interests.

\section{Publisher's Note}

Springer Nature remains neutral with regard to jurisdictional claims in published maps and institutional affiliations.

\section{Author details \\ ${ }^{1}$ Department of Experimental Psychology and Helmholtz Institute, Faculty of Social and Behavioural Sciences, Utrecht University, Utrecht, The Netherlands. ${ }^{2}$ Altrecht Academic Anxiety Centre, Utrecht, The Netherlands. ${ }^{3}$ Department of Psychiatry, Amsterdam Public Health Research Institute, VU University Medical Center and GGZinGeest, Amsterdam, The Netherlands. ${ }^{4}$ University Center Psychiatry, University Medical Center Groningen, Groningen, The Netherlands. ${ }^{5}$ Department of Clinical Psychology, Faculty of Social and Behavioural Sciences, Utrecht University, Utrecht, The Netherlands. ${ }^{6}$ Rob Giel Research Center \& Department of Psychiatry, University Medical Center Groningen, Groningen, The Netherlands. ${ }^{7}$ Department of Pharmaceutical Sciences, Division of Pharmacology, UIPS, Utrecht University, Utrecht, The Netherlands.}

Received: 6 March 2018 Accepted: 11 January 2019

Published online: 13 February 2019

\section{References}

1. Kessler RC, Angermeyer M, Anthony JC, DE Graaf R, Demyttenaere K, Gasquet I, et al. Lifetime prevalence and age-of-onset distributions of mental disorders in the World Health Organization's world mental health survey initiative. World Psychiatry. 2005;6(3):168-76.

2. Alonso J, Angermeyer MC, Bernert S, Bruffaerts R, Brugha TS, Bryson H, et al. Prevalence of mental disorders in Europe: results from the European study of the epidemiology of mental disorders (ESEMeD) project. Acta Psychiatr Scand Suppl. 2004;109(s420):21-7.

3. Gustavsson A, Svensson M, Jacobi F, Allgulander C, Alonso J, Beghi E, et al Cost of disorders of the brain in Europe 2010. Eur Neuropsychopharmacol. 2011;21(10):718-79.

4. Souětre $E$, Lozet $H$, Cimarosti I, Martin P, Chignon JM, Adés J, et al. Cost of anxiety disorders: impact of comorbidity. J Psychosom Res. 1994;38:151-60.

5. Klein Hofmeijer-Sevink M, Batelaan NM, Van Megen HJGM, Penninx BW, Cath DC, Van Den Hout MA, et al. Clinical relevance of comorbidity in anxiety disorders: a report from the Netherlands study of depression and anxiety (NESDA). J Affect Disord. 2012;137(1):106-12.

6. Sareen J, Cox BJ, Afifi, TO, de Graaf R, Asmundson GJG, ten Have M, Stein M. Anxiety disorders and risk for suicidal ideation and suicide attempts. Arch Gen Psychiatry. 2005;62(11):1249-57.

7. Hendriks SM, Spijker J, Licht CMM, Beekman ATF, Penninx BWJH. Two-year course of anxiety disorders: different across disorders or dimensions? Acta Psychiatr Scand. 2013;128(3):212-21.
8. Gloster AT, Hauke C, Höfler M, Einsle F, Fydrich T, Hamm A, et al. Long-term stability of cognitive behavioral therapy effects for panic disorder with agoraphobia: a two-year follow-up study. Behav Res Ther. 2013;51(12):830-9.

9. Donovan MR, Glue P, Kolluri S, Emir B. Comparative efficacy of antidepressants in preventing relapse in anxiety disorders - a meta-analysis. J Affect Disord. 2010;123(1):9-16.

10. Batelaan NM, Bosman RC, Muntingh A, Scholten WD, Huijbregts KM, van Balkom AJLM. Risk of relapse after antidepressant discontinuation in anxiety disorders, obsessive-compulsive disorder, and post-traumatic stress disorder: systematic review and meta-analysis of relapse prevention trials. BMJ. 2017:j3927.

11. Feske U, Chambless DL. Cognitive behavioral versus exposure only treatment for social phobia: a meta-analysis. Behav Ther. 1995;26(4):695-720.

12. Singewald N, Schmuckermair C, Whittle N, Holmes A, Ressler KJ. Pharmacology of cognitive enhancers for exposure-based therapy of fear, anxiety and trauma-related disorders. Pharmacol Ther. 2015;149:150-90.

13. Hofmann SG. Enhancing exposure-based therapy from a translational research perspective. Behav Res Ther. 2008;45(9):1987-2001.

14. Hofmann SG. Cognitive processes during fear acquisition and extinction in animals and humans: implications for exposure therapy of anxiety disorders. Clin Psychol Rev. 2008;28(2):199-210.

15. Duits P, Cath DC, Lissek S, Hox JJ, Hamm AO, Engelhard IM, et al. Updated meta-analysis of classical fear conditioning in the anxiety disorders. Depress Anxiety. 2015;32(4):239-53

16. Duits P, JMP B, Engelhard IM, Richter J, Huisman-van Dijk HM, Limberg A, et al. Latent fear conditioning trajectories in anxiety disorders and their predictive value on treatment outcome. Manuscr Prep. 2018.

17. Marsicano G, Wotjak CT, Azad SC, Bisogno T, Rammes G, Cascio MG, et al. The endogenous cannabinoid system controls extinction of aversive memories. Nature. 2002;418(6897):530-4.

18. Riedel G, Davies SN. Cannabinoid Function in Learning, Memory and Plasticity. Cannabinoids. Springer-Verlag: Berlin/Heidelberg; 2005. p. 445-77.

19. Berardi A, Schelling G, Campolongo P. The endocannabinoid system and post traumatic stress disorder (PTSD): from preclinical findings to innovative therapeutic approaches in clinical settings. Pharmacol Res. 2016;111:668-78.

20. Heitland I, Klumpers F, Oosting RS, Evers DJJ, Leon Kenemans J, Baas JMP. Failure to extinguish fear and genetic variability in the human cannabinoid receptor 1. Transl Psychiatry. 2012;2(9):e162.

21. Rabinak CA, Angstadt M, Sripada CS, Abelson JL, Liberzon I, Milad MR, et al. Cannabinoid facilitation of fear extinction memory recall in humans. Neuropharmacology. 2013;64:396-402.

22. Rabinak CA, Angstadt M, Lyons M, Mori S, Milad MR, Liberzon I, et al. Cannabinoid modulation of prefrontal-limbic activation during fear extinction learning and recall in humans. Neurobiol Learn Mem. 2014;113:125-34.

23. Das RK, Kamboj SK, Ramadas M, Yogan K, Gupta V, Redman E, et al. Cannabidiol enhances consolidation of explicit fear extinction in humans. Psychopharmacology. 2013;226(4):781-92.

24. Klumpers F, Denys D, Kenemans JL, Grillon C, Van Der Aart J, Baas JMP. Testing the effects of Delta9-THC and D-cycloserine on extinction of conditioned fear in humans. J Psychopharmacol. 2012:26(4):471-8.

25. Blessing EM, Steenkamp MM, Manzanares J, Marmar CR. Cannabidiol as a potential treatment for anxiety disorders. Neurotherapeutics. 2015;12(4):825-36.

26. Campos AC, Moreira FA, Gomes FV, Del Bel EA, Guimarães FS. Multiple mechanisms involved in the large-spectrum therapeutic potential of cannabidiol in psychiatric disorders. Philos Trans R Soc Lond Ser B Biol Sci. 2012;367(1607):3364-78.

27. Ahn K, McKinney MK, Cravatt BF. Enzymatic pathways that regulat endocannabinoid signaling in the nervous system. Chem Rev. 2008;108(5):1687-707.

28. Bitencourt RM, Pamplona FA, Takahashi RN. Facilitation of contextual fear memory extinction and anti-anxiogenic effects of AM404 and cannabidiol in conditioned rats. Eur Neuropsychopharmacol. 2008;18(12):849-59.

29. Stern C. A J, Gazarini L, Takahashi RN, Guimarães FS, Bertoglio LJ. On disruption of fear memory by reconsolidation blockade: evidence from Cannabidiol treatment. Neuropsychopharmacology. 2012;37(9):2132-42.

30. Duits P, Richter J, Baas JMP, Engelhard IM, Limberg-Thiesen A, Heitland I, et al. Enhancing effects of contingency instructions on fear acquisition and extinction in anxiety disorders. J Abnorm Psychol. 2017;126(4):378-91.

31. Dincheva I, Drysdale AT, Hartley CA, Johnson DC, Jing D, King EC, et al. FAAH genetic variation enhances fronto-amygdala function in mouse and human. Nat Commun. Nature Publishing Group. 2015;6:6395.

32. Lester KJ, Eley TC. Therapygenetics: using genetic markers to predict response to psychological treatment for mood and anxiety disorders. Biol Mood Anxiety Disord. 2013;3(1):4 
33. First MB, Spitzer RL, Gibbon M, Williams JBW. Structured clinical interview for DSM-IV-TR Axis I disorders, research version, patient edition. (SCID-I/P). New York: Biometrics Research, New York State Psychiatric Institute; 2002.

34. Schmand B, Lindeboom J, van Harskamp F. De Nederlandse leestest voor volwassenen. Lisse: Swets \& Zeitlinger; 1992.

35. Hoekstra RA, Bartels M, Cath DC, Boomsma DI. Factor structure, reliability and criterion validity of the autism-spectrum quotient (AQ): a study in Dutch population and patient groups. J Autism Dev Disord. 2008;38(8): 1555-66.

36. Rothbaum BO, Price M, Jovanovic T, Norrholm SD, Gerardi M, Dunlop B, et al. A randomized, double-blind evaluation of D-Clycoserine or alprazolam combined with virtual reality exposure therapy for posttraumatic stress disorder in Iraq and Afghanistan war veterans. Am J Psychiatry. 2014; 171(June):640-8

37. Norberg MM, Krystal JH, Tolin DF. A meta-analysis of D-Cycloserine and the facilitation of fear extinction and exposure therapy. Biol Psychiatry. 2008; 63(12):1118-26.

38. Faul F, Erdfelder E, Lang A-G, Buchner A. G*power: a flexible statistical power analysis program for the social, behavioral, and biomedical sciences. Behav Res Methods. 2007;39(2):175-91.

39. Hofmann SG, DiBartolo PM. Social anxiety: clinical, developmental, and social perspectives. New York, NY: Elsevier/Academic Press; 2010.

40. Kampman M, Keijsers G, Hendriks G. Behandeling Paniekstoornis. Amsterdam: Uitgeverij Boom; 2012

41. Englund A, Morrison PD, Nottage J, Hague D, Kane F, Bonaccorso S, et al. Cannabidiol inhibits THC-elicited paranoid symptoms and hippocampaldependent memory impairment. J Psychopharmacol. 2013;27(1):19-27.

42. Marks I, Mathews A. Brief standard self-rating for phobic patients. Behav Res Ther. 1979;17(3):263-7.

43. Beck AT, Epstein N, Brown G, Steer RA. Others. An inventory for measuring clinical anxiety: psychometric properties. J Consult Clin Psychol. 1988;56(6):893-7.

44. Beck AT, Steer RA, Brown GK. Beck depression inventory-II. San Antonio. 1996;78(2):490-8.

45. Chambless DL, Caputo GC, Bright P, Gallagher R. Assessment of fear of fea in agoraphobics: the body sensations questionnaire and the agoraphobic cognitions questionnaire. J Consult Clin Psychol. 1984 Dec;52(6):1090-7.

46. Turner SM, Beidel DC, Dancu CV, Stanley MA. An empirically derived inventory to measure social fears and anxiety: the social phobia and anxiety inventory. Psychol Assess A J Consult Clin Psychol. 1989;1(1):35-40.

47. de Vente W, Majdandžić M, Voncken MJ, Beidel DC, Bögels SM. The SPAI-18, a brief version of the social phobia and anxiety inventory: reliability and validity in clinically referred and non-referred samples. J Anxiety Disord. 2014;28(2):140-7.

48. Busner J, Targum SD. The clinical global impressions scale: applying a research tool in clinical practice. Psychiatry (Edgmont). 2007;4(7):28-37.

49. Wolpe J. The practice of behavior therapy. New York: Pergamon Press; 1969.

50. Guy W. ECDEU assessment manual for psychopharmacology. Education and Welfare: U.S. Department of Health; 1976.

51. Benito KG, Walther M. Therapeutic process during exposure: habituation model. J Obsessive Compuls Relat Disord. 2015;6:147-57.

52. Shear MK, Brown TA, Psy D, Barlow DH, Ph D, Money R, et al. Multicenter collaborative panic disorder severity scale. Am J Psychiatry. 1997;154(11):1571-5.

53. Chambless DL, Caputo GC, Jasin SE, Gracely EJ, Williams C. The mobility inventory for agoraphobia. Behav Res Ther. 1985;23(1):35-44.

54. Mennin DS, Fresco DM, Heimberg RG, Schneier FR, Davies SO, Liebowitz MR. Screening for social anxiety disorder in the clinical setting: using the Liebowitz social anxiety scale. J Anxiety Disord. 2002;16(6):661-73.

55. Bouman TK. De Agoraphobic Cognition Questionnaire (ACQ). Gedragstherapie. 1995;28:301

56. Steer RA, Ranieri WF, Beck AT, Clark DA. Further evidence for the validity of the Beck anxiety inventory with psychiatric outpatients. J Anxiety Disord. 1993;7(3):195-205.

57. van der Does A. Manual for the Dutch version of the Beck depression inventory-second edition (BDI-II-NL). Amsterdam, NL: Harcourt; 2002.

58. Bouman TK. De Body Sensations Questionnaire (BSQ). Gedragstherapie. 1998:31:165.

59. Van Zuuren FJ. The Fear Questionnaire. Some data on validity, reliability and layout. Br J Psychiatry. 1988;153(NOV.):659-662.

60. Baker SL, Heinrichs N, Kim HJ, Hofmann SG. The Liebowitz social anxiety scale as a self-report instrument: a preliminary psychometric analysis. Behav Res Ther. 2002;40(6):701-15.
61. Shear K, Rucci P, Williams J, Frank E, Grochocinski V, Vander Bilt J, et al. Reliability and validity of the panic disorder severity scale: replication and extension. J Psychiatr Res. 2001;35(5):293-6.

62. Forkmann T, Scherer A, Boecker M, Pawelzik M, Jostes R, Gauggel S. The clinical global impression scale and the influence of patient or staff perspective on outcome. BMC Psychiatry BioMed Central Ltd. 2011;11(1):83.

63. Bernstein DP, Fink L. Childhood trauma questionnaire: a retrospective selfreport manual. San Antonio, TX: The Psychological Corporation; 1998.

64. Leweke FM, Piomelli D, Pahlisch F, Muhl D, Gerth CW, Hoyer C, et al. Cannabidiol enhances anandamide signaling and alleviates psychotic symptoms of schizophrenia. Transl Psychiatry. 2012;2(3):e94.

65. Bouwmans C, De Jong K, Timman R, Zijlstra-Vlasveld M, Van der FeltzCornelis C, Tan Swan S, et al. Feasibility, reliability and validity of a questionnaire on healthcare consumption and productivity loss in patients with a psychiatric disorder (TiC-P). BMC Health Serv Res. 2013;13:217.

66. Hakkaart-van Roijen L, Van der Linden N, Bouwmans C, Kanters T, Tan SS. Kostenhandleiding: Methodologie van kostenonderzoek en referentieprijzen voor economische evaluaties in de gezondheidszorg. Zorginstituut Nederland: Diemen; 2015.

67. EuroQolGroup. EuroQol--a new facility for the measurement of healthrelated quality of life. Health Policy (New York). 1990;16(3):199-208.

68. Hofmann SG. Schrödinger's cat and d-Cycloserine to augment exposure therapy—both are alive and dead. JAMA Psychiatry. 2016;73(8):771.

69. Bergamaschi MM, Queiroz RHC, Chagas MHN, de Oliveira DCG, De Martinis BS, Kapczinski F, et al. Cannabidiol reduces the anxiety induced by simulated public speaking in treatment-naïve social phobia patients. Neuropsychopharmacology. 2011;36(6):1219-26.

70. Foa EB, Kozak MJ, Barlow D, Cuthbert B, Foa U, Hallam D, et al. Emotional processing of fear: exposure to corrective information. Psychol Bull. 1986; 99(1):20-35
Ready to submit your research? Choose BMC and benefit from:

- fast, convenient online submission

- thorough peer review by experienced researchers in your field

- rapid publication on acceptance

- support for research data, including large and complex data types

- gold Open Access which fosters wider collaboration and increased citations

- maximum visibility for your research: over $100 \mathrm{M}$ website views per year

At BMC, research is always in progress.

Learn more biomedcentral.com/submissions 